\title{
Electro-Pneumatic System Monitoring Using Multivariate Latent Methods
}

\author{
M. Ola*, W.M. Hussein*, I.A. El-Sherif* and I. Saleh*
}

\begin{abstract}
Pneumatic actuators exhibit highly nonlinear characteristics due to air compressibility, significant friction presence and the nonlinearities of control valves. Monitoring by acquiring the system's transfer function accurately can be difficult for nonlinear systems. This paper outlines the use of a statistical multivariate technique called Principal Component Analysis (PCA) and Projection to Latent Structure Discriminant Analysis (PLS-DA) and applies them to the monitoring of electro-pneumatic system. This approach is used to integrate system sensory using data (Pressure, displacement and vibration) from a printing machine, an electro-pneumatic system. Experiments were conducted under three conditions, normal, throttled and leaked system. The models were tested under different conditions with the experimental results, showing that the proposed technique can be used for electro-pneumatic system monitoring in a real-time environment and could successfully differentiate between new process conditions.
\end{abstract}

Keywords: Electro-pneumatic system monitoring, Electro-pneumatic system, Multivariate analysis, PCA, PLS-DA.

\section{Introduction}

In recent printing industry, on-line monitoring of process performance is extremely important for printing machines safety, production efficiency and product quality. Printing machines are consisting of very expensive parts. They include many of pneumatic systems which have various pneumatic parts like compressor, accumulators, valves, actuators and other pneumatic elements. As the mechanical condition of the machine is changed, due to variation of its technical state, the changes in operating environments, throttled state, leakage state, etc., result in variation in its performance. Vibration in printing machines is caused by air flow and mechanical parts ...... etc. Vibration analysis is most commonly used for machine condition monitoring [1]. In addition the pressure and displacement analysis is discussed in [2,3]. A generic fault detection and diagnosis approach for pneumatic driven railway assets was made but with no dynamic analysis [4]. Discussion of the properties of the TSK/Mamdani approaches (Takagi and Sugeno, 1985; Sugeno and Kang, 1988 and Mamdani (1995, 1996) combined with NN learning algorithms) and neuro-fuzzy (NF) fault diagnosis within an application study of an electro-pneumatic valve actuator in a sugar factory [5]. Also there is an approach for process monitoring using a multivariate statistical technique, namely kernel principal component analysis is studied on an electro-pneumatic valve actuator [6]. In this work, a statistical multivariate technique called Principal Component Analysis (PCA) and Projection to Latent Structure Discriminant Analysis (PLS-DA) is applied to a Printmaster

\footnotetext{
* Egyptian Armed Forces.
} 
QM 46 type of printing machine. As health monitoring techniques in order to predict the machine faults prior appearing to save effort and money spent through traditional techniques used for machine monitoring. The pneumatic system of the printing machine under investigation, as shown in figure 1, consists of an electric motor with compressor, accumulator, electro pneumatic directional control valve and the cylinder. This cylinder connects the plate cylinder to the blanket cylinder of the printing machine.

Most monitoring techniques presently available describe the variation of one or a limited number of machine components using a heuristic approach which gives limited information about machine condition. Among these techniques, artificial neural network ANNs and its combination with other methods have been the most predominant to date $[7,8]$. ANNs techniques are characterized as black box approaches which model the relation between different variables to a desired response without giving any information about what happens inside the process.

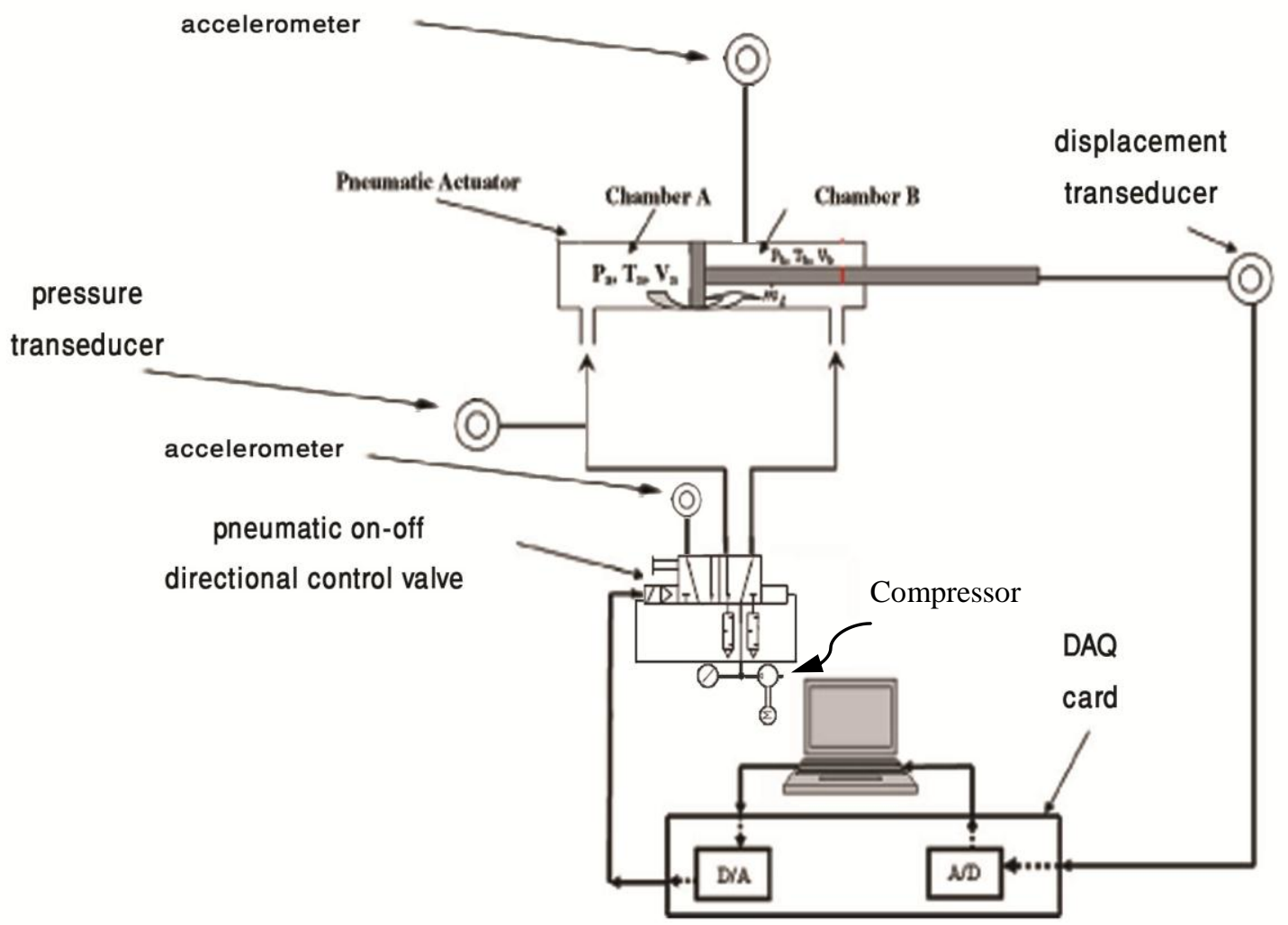

Fig. 1 Scheme drawing for Printmaster QM 46 pneumatic system

Also some of these approaches are susceptible to missing data due to sensor reliability issues, and some applications use many charts to monitor individual process. In addition, issues associated with collinearity and dimensionality needs to still be specifically addressed in these techniques. Looking for a generic approach to solve many of these limitations can be addressed through the use of multivariate statistical analysis, a data driven modeling approach [9]. Multivariate statistical process control (MSPC) takes a different approach as compared to the other methods mentioned previously; the most fundamental difference with this approach is that the model is based on non-causal empirical correlations extracted from normal plant operating data when only common cause variation exists [10]. The simplicity of this approach is that there is no need for a fundamental model of the system and only data from normal operation needs to be used, which is generally available in some form at most machines. Among the approaches used in multivariate analysis are: a projection method which called 
(PCA) $[11,12]$ and projection to latent structure (PLS) [13]. Many applications of these two techniques have been successfully applied in other fields of process monitoring [7, 14-19] ranging from batch to continuous processes. (PCA) is the most widely used data-driven technique for process monitoring which has been heavily studied and applied to industrial systems over the past decade. PCA is an optimal dimensionality reduction technique in terms of capturing the variance of the data, and it accounts for correlations among variables. The lower-dimensional representations of the data produced by PCA can improve the proficiency of detecting and diagnosing faults using multivariate statistics. The principal components span a low dimensional subspace used for analysis. The details of linear PCA can be found elsewhere [20].

This paper outlines the use of multivariate projection approach to develop a framework to monitor the printing machine under study. By integrating different data collected from different accelerometers, pressure, displacement transducers fixed at different machine positions under different operating pressures and conditions. Models based on PCA and PLSDA can be developed into a single user interface at which, machine performance can be monitored using the latent space of these models.

\section{Multivariate PCA and PLS-DA Basic Theory}

\section{a. Multivariate PCA theory:}

PCA decomposes the variance and covariance structure of a data matrix by defining linear combinations of the columns in the original matrix. Moreover, PCA extracts information from data sets by computing a smaller data set and other summary information that adequately captures most of the underlying features from the larger data set. The point that needs to be stressed is that the data can be reduced to a size which is more manageable but contains the features that are often of interest. PCA extracts a score matrix, T, and a loading matrix, P, from $\mathrm{X}$. These matrices have the following dimensions:

$$
X: N \times K \quad T: N \times A \quad P: K \times A
$$

We call the first column of $\mathrm{T}$ and $\mathrm{P}$ by their shorter forms, $\mathrm{t}_{1}$ and $\mathrm{p}_{1}$ respectively. The lower case letters indicate that these are vectors, upper case letters indicate matrices. Extracting only one principal component (that is, a single score vector and loading vector) gives:

$\mathrm{X}=\mathrm{t}_{1} p_{1}^{T}+\mathrm{E} 1 \quad$ and extracting a second principal component: $\mathrm{X}=\mathrm{t}_{1} p_{1}^{T}+\mathrm{t}_{2} p_{2}^{T}+\mathrm{E} 2[12,21]$

We continue in this manner until we extract principal components and then group these score and loading vectors to form matrices $\mathrm{T}$ and $\mathrm{P}$ :

$$
\mathrm{T}=\left[\mathrm{t}_{1} \mathrm{t}_{2} \ldots \mathrm{t}_{\mathrm{A}}\right] \text { and } \mathrm{P}=\left[\mathrm{p}_{1} \mathrm{p}_{2} \ldots \mathrm{p}_{\mathrm{A}}\right]
$$

The eigenvectors of the real symmetric matrix $\mathrm{X}^{\mathrm{T}} \mathrm{X}$ give us exactly the loading matrix $\mathrm{P}$. This is the loading matrix obtained by extracting all principal components. Once we have $\mathrm{P}$ we use the last line of the following relationship to compute T:

$$
\mathrm{TP}^{\mathrm{T}}=\mathrm{X} \quad \mathrm{TP}^{\mathrm{T}} \mathrm{P}=\mathrm{XP} \quad \mathrm{T}=\mathrm{XP}
$$

A more in-depth discussion, which also highlights some geometric concepts of PCA, can be found in [11]. The algorithm used to calculate the PCA is the NIPALS (Nonlinear Iterative Partial Least Squares) algorithm [12,21]. 


\section{b. Multivariate PLS-DA Theory}

The PLS-DA aims to find a line or a plane that separates classes of observations based on their values, The PLS-DA model is based on building classes or clusters using pre-knowledge about existing groups (supervised learning). The model is established using a set of experimental runs (training set). PLS-DA works on two matrices, X-matrix as input and Y-matrix as output. The X-matrix contains all the data (i.e. sensory data) with all the known groups (i.e. normal and faulty states). The Y-matrix contains dummy variables in columns equal to the number of already known class of data. In the $\mathrm{Y}$-matrix we assign to each observation from the same group a code number constant for the entire group. For example, in case we have three groups to cluster, the first group (e.g. sharp tool data) is assigned with a code value of " $1,0,0$ " for each row (observation) of the same group. Depending on the number of samples or observations from each group, there will be an equal number of code values with the same value " $1,0,0$ ". The second group will take another code of " $0,1,0$ ", and so on for other groups (i.e. $0,0,1$ etc...). In this way the approach tries to model X-Y variables by finding the latent directions that best separates the known groups. In other words, sometimes factors are introduced that describe the data from different groups. So, we must assign some distinct levels to these factors to help discriminate between different groups. We call these factors qualitative or dummy variables. Mathematically this can be illustrated by adding new variables " $G$ " to the model with some regression coefficients say "c", and set $G=1$ for data from one group and $G=0$ for data from another group in the following way: $\mathrm{Y}=\mathrm{f}+(\mathrm{c} * \mathrm{G})$. Suppose that (f) represents the fitted model, so when $\mathrm{G}=0$, the data from the first group is highlighted: $\mathrm{Y}=\mathrm{f}$ and by putting $\mathrm{G}=1$, the data from the second group is highlighted: $\mathrm{Y}=\mathrm{f}+\mathrm{c}$ where $\mathrm{c}$ estimates the difference in levels between the responses of two groups of data. In this way the data from different groups will be clustered depending on their regression coefficient values $[13,16]$.

\section{Experimental Setup}

Figure 2 shows the schematic of the experimental setup used to validate the proposed method. The main feature of the test rig is to perform integrated components of mechanical, electronics, and computer interface structure, with high computational capacity and software. The position of the pneumatic cylinder, of the servo-system, presents the system characteristics depending on its variation. The pneumatic unit consists of a compressor, 5/2 directional control valve with on-off solenoid which operating at voltage $23 \mathrm{~V}$, a cylinder has stroke length of $52.5 \mathrm{~mm}$. The sensors are used to monitor the system mentioned in Table 1 shown below:

Table 1 The sensors are used to monitor the system

\begin{tabular}{c|c|c}
\hline \hline No. & Name & Specification \\
\hline \hline 1 & $\begin{array}{c}\text { Linear variable differential transformer } \\
\text { (LVDT) }\end{array}$ & $\begin{array}{c}\text { A voltage range source } 0 \text { to } 10 \mathrm{~V}, \mathrm{a} \\
\text { displacement range from } 0 \text { to } 100 \mathrm{~mm}, \\
\text { sensitivity } 9.5 \mathrm{~V}+/-0.05 \% .\end{array}$ \\
\hline 2 & Pressure transducer & $\begin{array}{c}\text { A voltage range source } 0 \text { to } 10 \mathrm{~V}, \mathrm{a} \\
\text { pressure range from } 0 \text { to } 10 \mathrm{bars} .\end{array}$ \\
\hline 3 & Non- magnetic accelerometer transducer & $\begin{array}{c}\mathrm{A} / 120 / \mathrm{V} \text { has a voltage sensitivity } \\
10 \mathrm{mV} / \mathrm{g} .\end{array}$ \\
\hline 4 & Non- magnetic accelerometer transducer & $\begin{array}{c}\text { ACC103 has a voltage sensitivity } \\
10 \mathrm{mV} / \mathrm{g} .\end{array}$ \\
\hline
\end{tabular}


All signals were sent to a computer via a National Instruments (NI) DAQ card PCI 4472 Dynamic Signal Analyzer (DSA) through an A/D converter terminal. The DAQ card has 8 channels with a maximum sampling rate of $102.4 \mathrm{kS} / \mathrm{s}$. The solenoid of the valve has been controlled by a digital output from the DAQ card. The testing algorithm has been achieved under control operation of the pneumatic system input pressure $4-6.4$ bar for the two states: normal and faulty cases using Labview software.

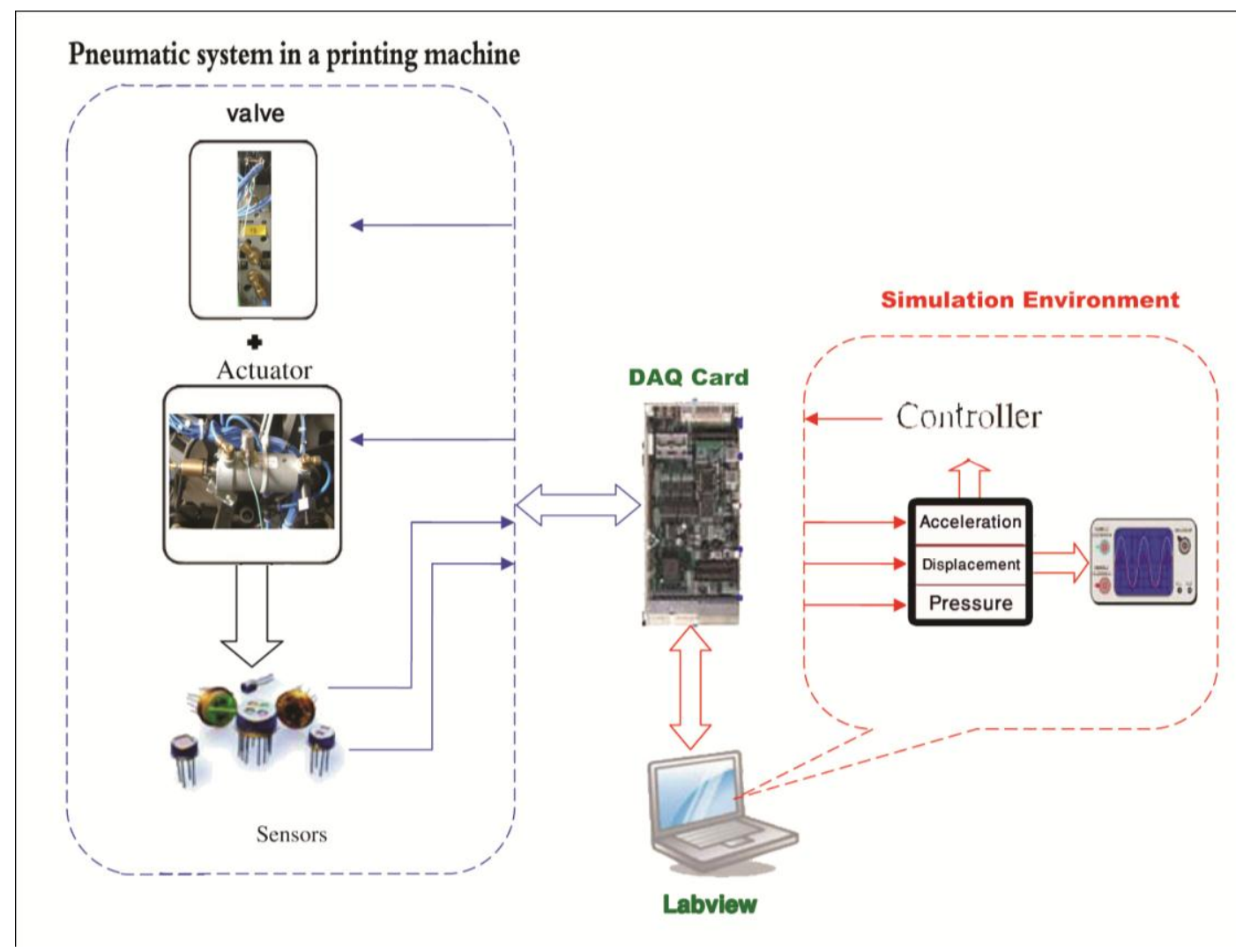

Fig. 2 Schematic of the experimental setup

\section{Model Building, Configuration and Results}

The multivariate PCA model is a linear model given in matrix form by: $X=\mathrm{TP}^{\mathrm{T}}+\mathrm{E}_{\mathrm{A}}$ $[12,21]$. However, there exist non-linear PCA versions even by augmenting the original matrix with the non-linear factors or by building a non-linear relation between score factors [12]. To build the model using process variables, let $X$ include variables containing the information in the sensory data. Figure 3 illustrates how the score plot is built for a simple case 3-variables and 2-scores. After determining the direction of maximum variation by iterative steps and get the second orthogonal direction by the same way after subtracting the first component, we rotate the new plane determined by the new score variables $t_{1}$ and $t_{2}$ and then monitor the movement of the process variables in the reduced dimensional space during printing.

A total of 4 variables were used to generate the input matrix " $X$ ". These variables represent the root mean square of the time domain records of the sensory signals (actuator displacement, system pressure, valve vibration and actuator vibration).

All of the models were built using SIMCA-P code developed by Umetrics based on PCA [9]. The PCA model is based on building classes or clusters using existing information inside the data (unsupervised learning). The model is established using a set of experimental runs called the training set that represents the normal operation of the printing machine. Finally, regression is made on the matrix X based on NIPALS algorithm. 


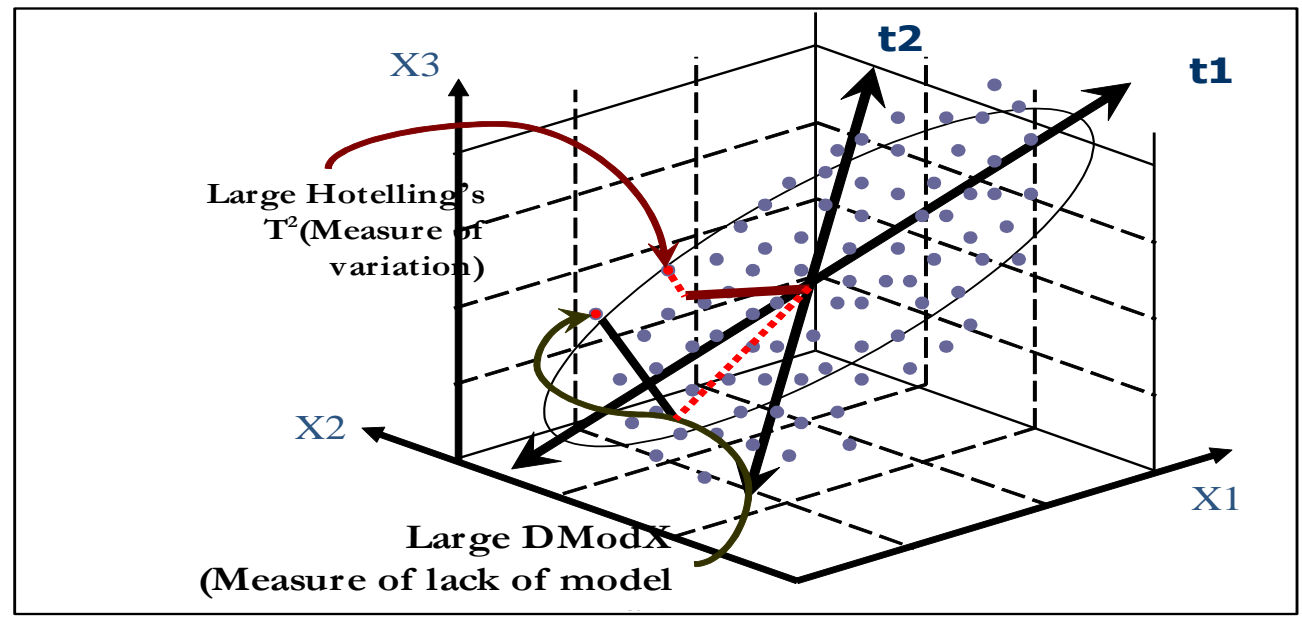

Fig. 3 Establishing score plot for three variables

To explore the collected data a first model using PCA was built using all the dataset by crossvalidation, now obtaining a two component model, with: R2X(cum): 0.81, Q2(cum) : -0.105 . It is seen that the model factor Q2cum, is very small, but it is still better to first get a general look at the whole dataset without removing any variable or observation. Figure 4 illustrates a scatter plot of the two score vectors ( $\mathrm{t} 1$ and $\mathrm{t} 2$ ) of the PCA model using a set of 10 observations. It provides a clear vision of the dispersion of the data, with the normal process data being discriminated against the faulty data along the first two components. This clustering is shown by a straight line separating the different conditions (normal operation of the electro-pneumatic system and different levels of leaked and throttled states of the system).

PCA-X Score Plot



Fig. 4 A scatter plot of the two score vectors (t1 and t2) of the PCA model

More analysis is made by examining the loading plot which shows the relation between the different variables. Figure 5 shows the loading plot clarifying the relation between the 4 variables. In addition, the score and loading plots are superimposed; this means that variables lying in each quarter of the loading plot are contributing to the changes between the observations in the score plot. So, by investigating the loading plot (figure 5), one can detect directly which variables are responsible or more affecting a specified group of data in the score plot. 


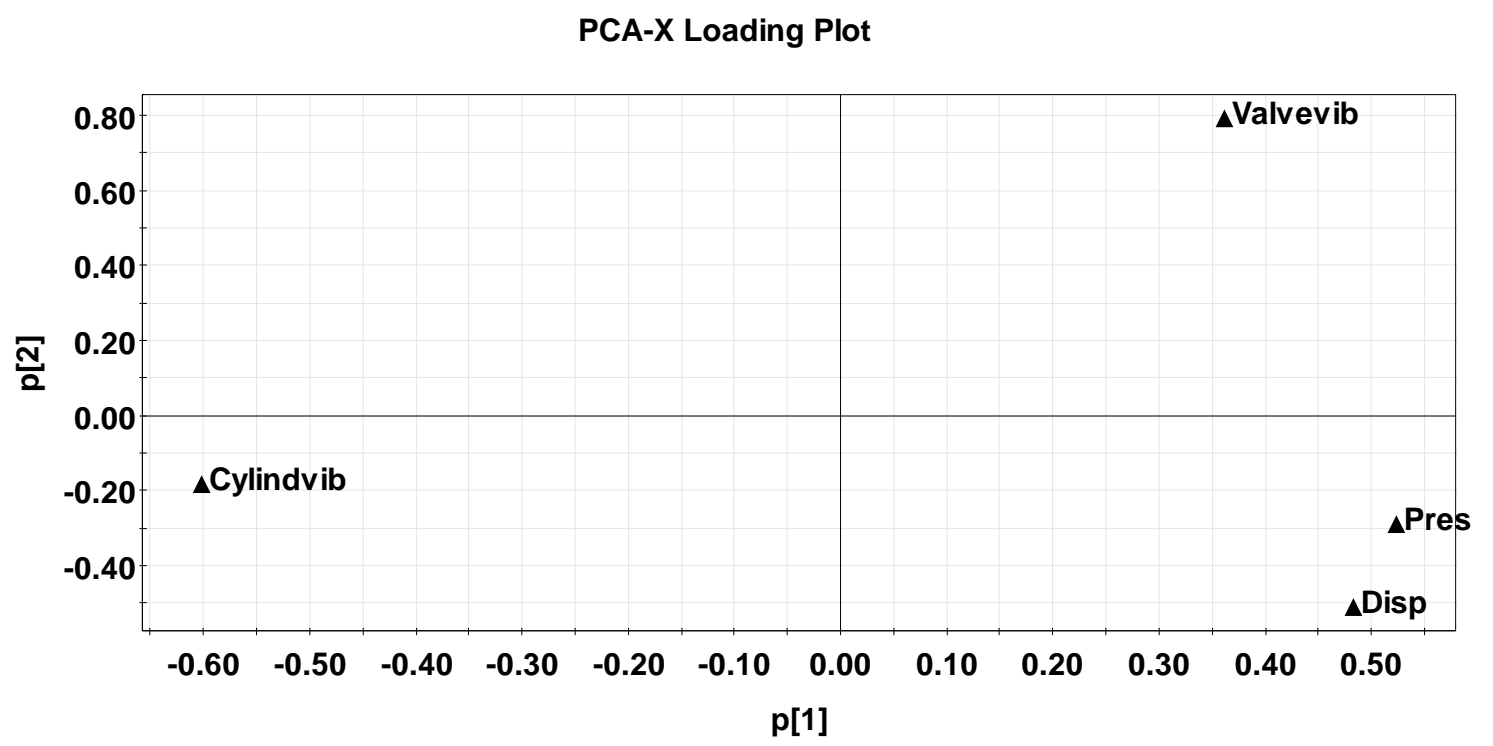

Fig. 5 PCA-X loading plot

From these two figures, one can notice, how pressure, displacement and valve vibration variables in the right corner of the loading plot contribute to the right swarm (faulty states) of data in the score plot. In other words, these variables are directly correlated to the faulty states while the cylinder vibration is inversely correlated with them.

More investigation carried out using control charts and contribution plots as shown in figure 6 to quickly assess which factors affect these different process shifts. Figure 6 shows the contribution plots for high throttle state.

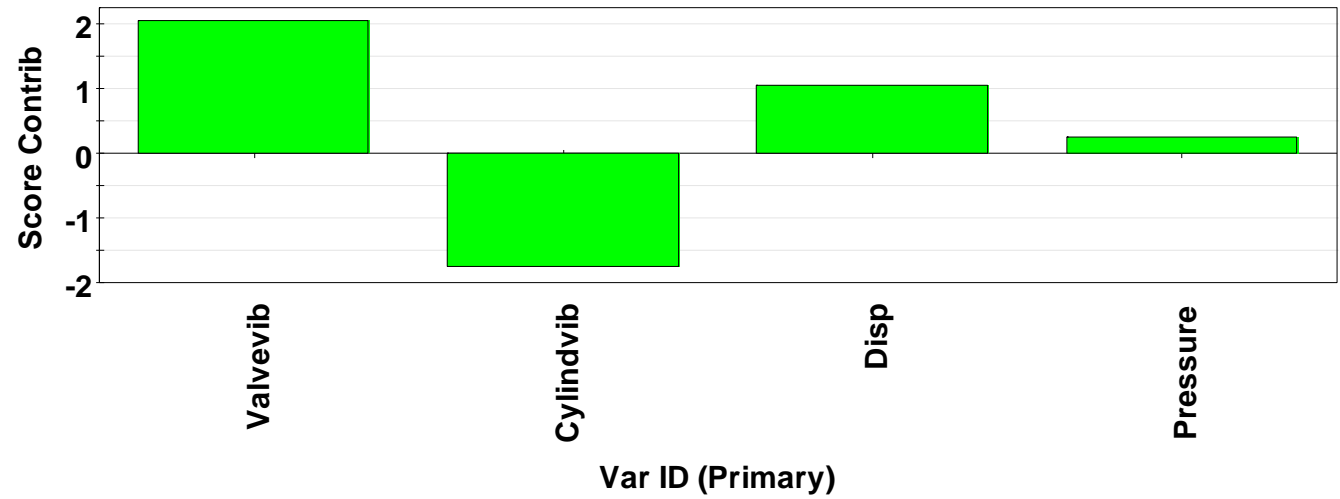

Fig. 6 Control chart for the contribution plots for high throttle state

One can notice that both valve and cylinder vibrations are responsible for this shift, alarming on the occurrence of high throttle.

The main target is to build a monitoring system to the printing machine. Also, the PCA approach is based on using available database that give an acceptable level of process quality to build the monitoring system and then the model can detect any fault or shift from normal operation. Therefore, the same setting is repeated again with the use of data of the normal process operation as a training set under different system pressure values. For better classification, another attempt has been made by augmenting the x-matrix with square terms between variables. It ends with 8 variables and is fitted by cross-validation, to get a two component model with: R2X (cum): 0.734, Q2 (cum): -0.21. Figure 7 illustrates a scatter plot of the two score vectors ( $\mathrm{t} 1$ and $\mathrm{t} 2$ ) of the PCA model using the normal process data. 


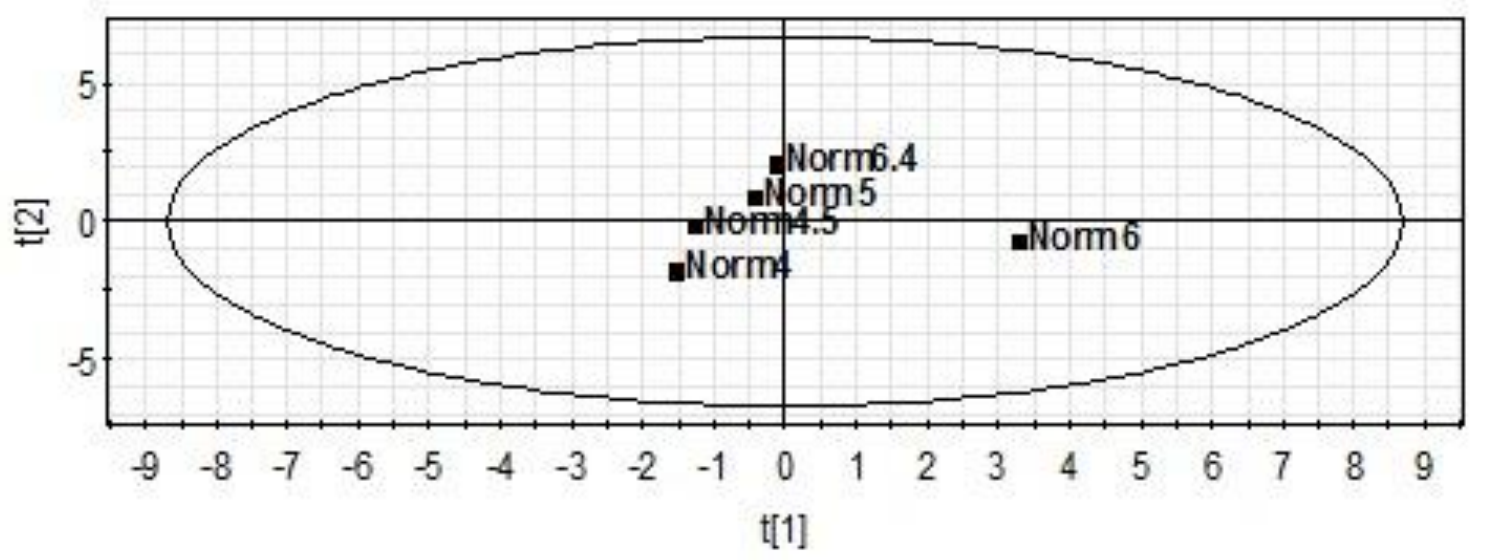

Fig. 7 A scatter plot of the two score vectors (t1 and t2) of the PCA model using the normal process data

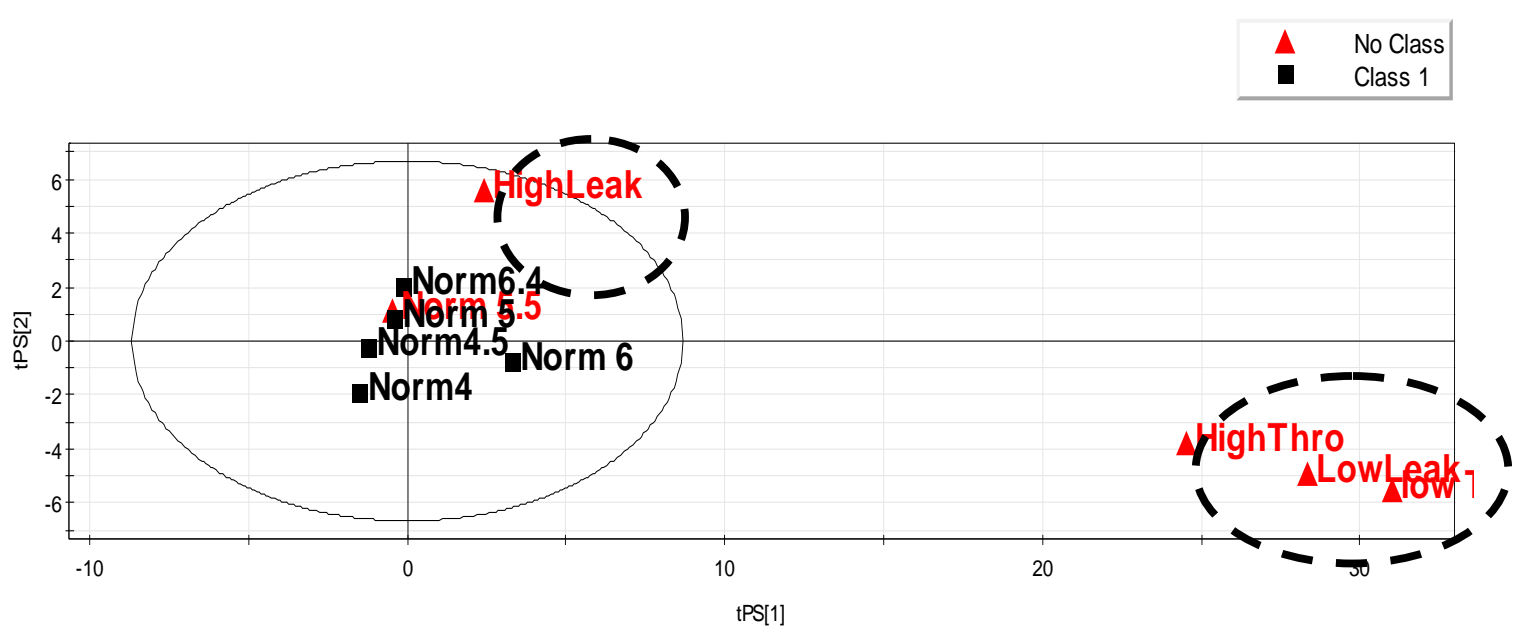

Fig. 8 The developed PCA model used for validation set

The developed PCA model was used on a validation set of the remaining 5 observations (1 normal at 5.5 bar, 4 faulty states: High and low throttle and high and low leakage). The PCA scores values $(\mathrm{t} 1, \mathrm{t} 2)$ for these test observations are indicated by triangle points and manually highlighted with dotted circles in the $\mathrm{t} 1 \mathrm{vs}$. $\mathrm{t} 2$ score plot in figure 8 . It can be seen from the figure that the PCA model succeeds in classifying the new observations normal, throttled and leaked data. One can notice that the high leakage state lies near the normal group as a result of the same data nature (low pressure).

More investigation carried out using control charts and contribution plots as shown in figure 9 to quickly assess which factors affect these different shifts showing the contribution plots for high throttled state. One can notice that cylinder vibration is responsible for this shift, alarming on the occurrence of throttled pneumatic system (high throttle results in low air pressure going to the cylinder, lowering system vibration level) and showing the Hotelling's $\mathrm{T}^{2}$ and DModX model residual plots with the test set. A clear advantage is realized from modeling the X-space over the present approaches. In case of the occurrence of high leakage (not modeled) but similar to the same normal process state, the Hotelling's $\mathrm{T}^{2}$ chart failed to detect this process shift but is alarmed through the model residual DModX.

The second model will be based on PLS-DA. Let X includes variables containing the information in the sensory data (Pressure, vibration, and displacement). Then, let Y consists of two dummy variables which code the two groups intended to separate: normal data, faulty states. Finally, regression is made between $\mathrm{X}$ and $\mathrm{Y}$ based on a superficially "similar" NIPALS (Nonlinear Iterative Partial Least Squares) algorithm. 
rms 1.M7 (PCA-X), Untitled, PS-rms1

Shewhart (Subgroup 1): T2PS[Comp. 1]

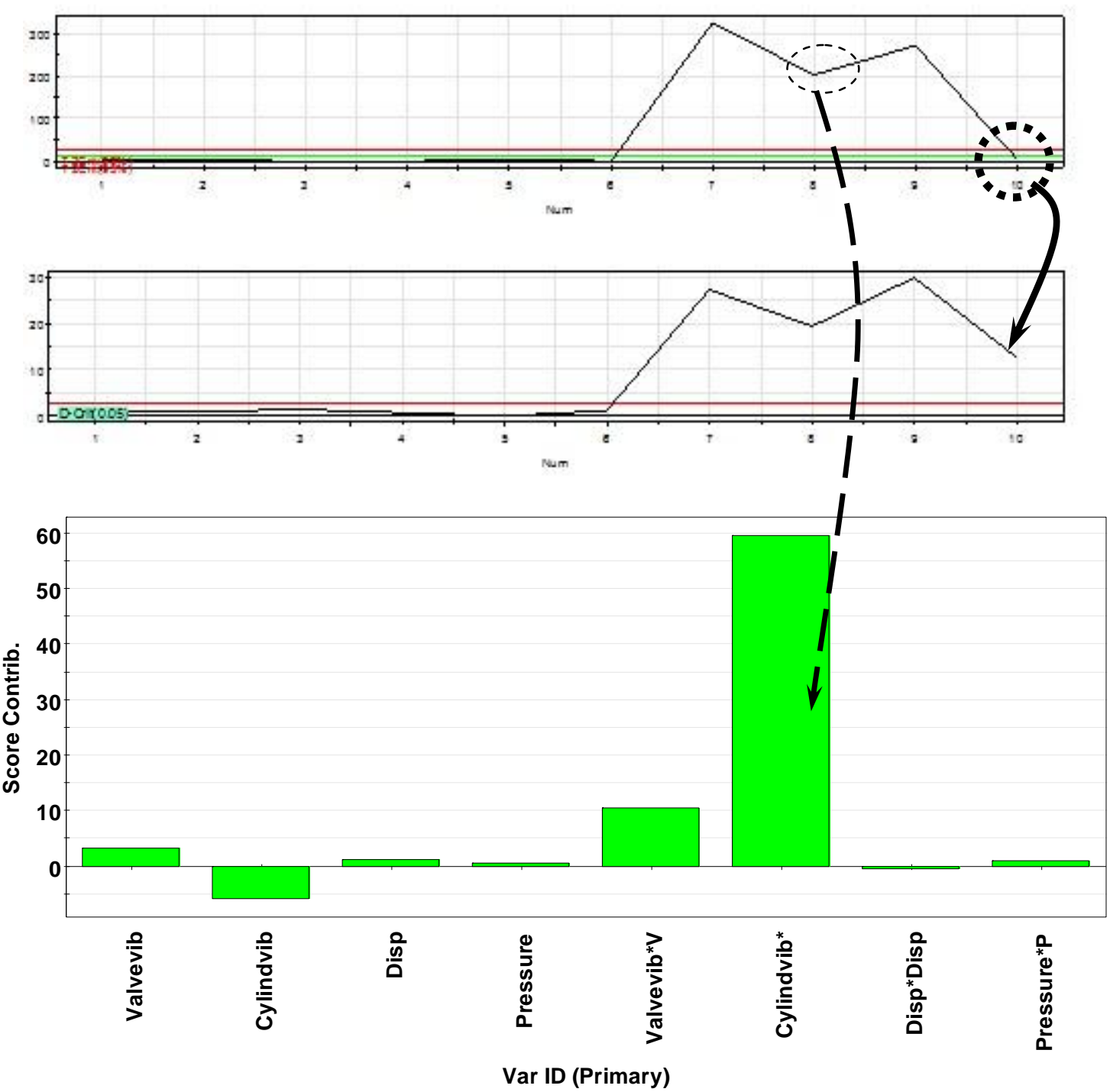

Fig. 9 The contribution plots for high throttled state

Out of the 10 observations ( 6 normal, 4 fault), a training set of 8 observations ( 5 normal, 3 fault) representing the two groups (normal, fault) were chosen to develop the PLS-DA regression model. The PLS-DA model developed was then tested on a subset of the remaining 2 observations ( 1 normal, 1 fault). The PLS-DA model was built on a training dataset by cross-validation, now obtaining a two components model, with: R2X (cum): 0.76, R2Y (cum): 0.92, Q2 (cum): 0.29. Figure 10 illustrates a scatter plot of the two score vectors (t1 and $\mathrm{t} 2$ ) of the trained PLS-DA model using the training set data. Solid box points represent the training set samples of the normal data group and solid stars represent the faulty data group. According to the manually highlighted point clusters in the figure (done only for visual purposes), it can be seen that the discrimination between normal and faulty data is very good. The figure provides a clear view of the dispersion in the data, with the normal data being discriminated against the faulty states data along the first component, due to the fact that now this is the one having the most discriminating power, because the PLS-DA model maximizes the covariance between the $\mathrm{X}$ and $\mathrm{Y}$ matrices, constituted by the different groups of data. 


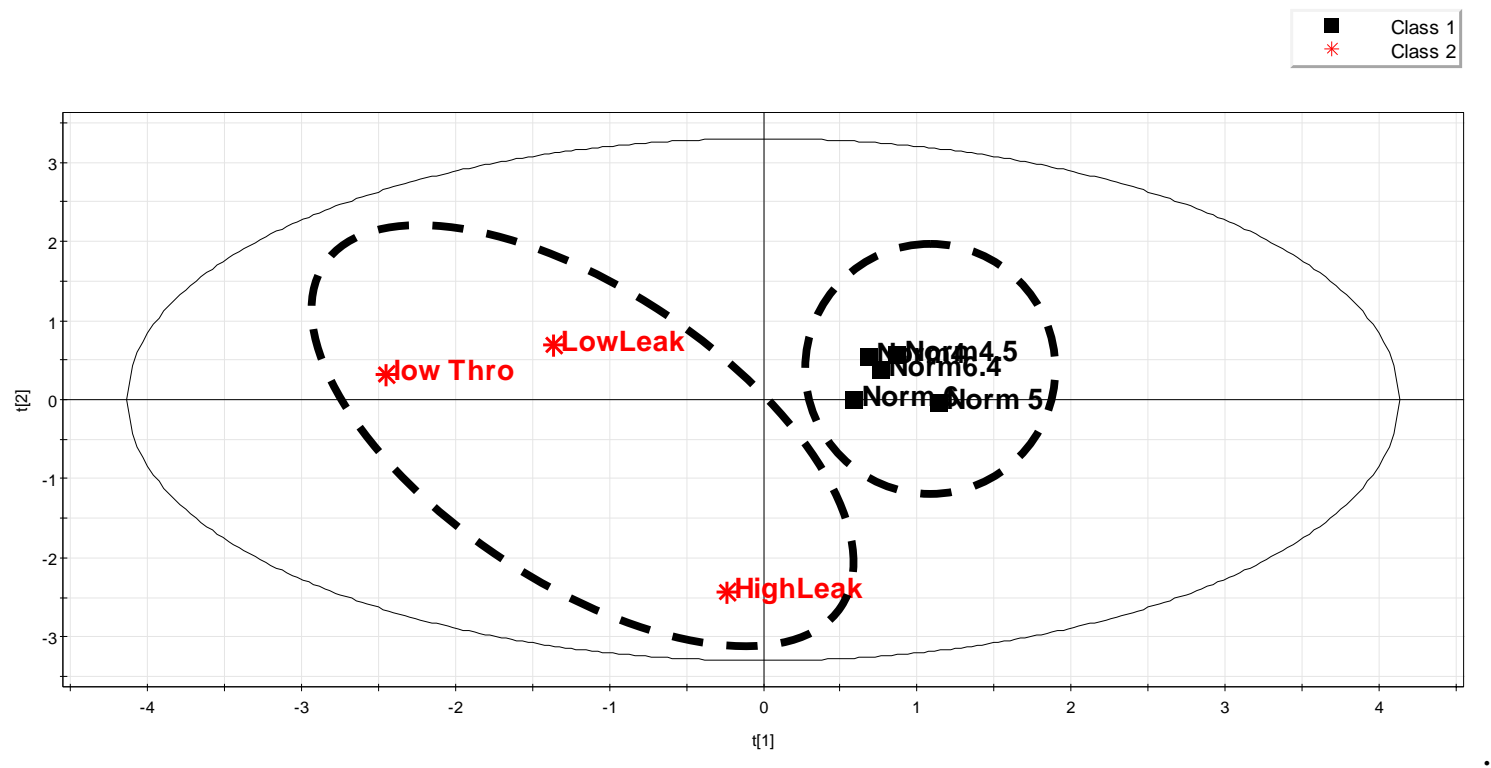

Fig. 10 A scatter plot of the two score vectors ( $t 1$ and $t 2)$ of the trained PLS-DA model using the training set data

A model developed from a training set must be validated and tested with new observations not included in the original training set. Hence, the PLS-DA model developed was used on a test set of the remaining 2 observations. The PLS-DA score values $(t 1, t 2)$ for these test observations are indicated by triangle points and manually highlighted with dotted circles in the $\mathrm{t} 1 \mathrm{vs} . \mathrm{t} 2$ score plot in figure 11 . It can be seen from the figure that the PLS-DA model succeeds in classifying the new observations normal, and high throttled data. The $\mathrm{t} 1-\mathrm{t} 2$ score of normal data is magnified to illustrate the classification of the tested observations.

\section{Conclusions}

Multivariate projection techniques are statistical methods, which can be used to describe, analyze and model multivariate data, while simultaneously measuring several predictors and response variables. The application of multivariate techniques to monitor an electro pneumatic system of a printing machine is the main contributions to the development of a generic machine monitoring, fault detection and optimization system. The approach uses experimental data for model building and testing. Multivariate approaches model the data in the X-space where the advantage of the contribution plots can be seen for machine monitoring. These plots facilitate the detection of the variables responsible for any condition change both those modeled or unmodeled. Through the use of a well selected system of sensors which augments the matrix of input variables (X-matrix) with key feature components from these signals, a machine components condition can be detected. 




Fig. 11 Validation and testing model of PLS-DA

\section{References}

[1] E. M. Said, W.M. Hussein, A.M. Salem and Mohamed K.I, "Using Multivariate Latent Models to Monitor a Printing Machine and Predict Machine Failure", Proceedings of the 12th International Conference on Aerospace Sciences \& Aviation Technology, Military Technical College, Cairo, Egypt, 2007.

[2] M. Demetgul, I.N. Tansel and S. Taskin, "Fault diagnosis of pneumatic systems with artificial neural network algorithms", Elsevier Ltd, M. Demetgul et al. Expert Systems with Applications 36, 2009.

[3] E. M. Said, W.M. Hussein, A.M. Salem and Mohamed K.I, "Using Multivariate Latent Models to Monitor a Printing Machine and Predict Machine Failure", Proceedings of the 12th International Conference on Aerospace Sciences \& Aviation Technology, Military Technical College, Cairo, Egypt, 2007.

[4] M. Demetgul, I.N. Tansel and S. Taskin, "Fault diagnosis of pneumatic systems with artificial neural network algorithms", Elsevier Ltd, M. Demetgul et al. Expert Systems with Applications 36, 2009. 
[5] Ž.Nakutis and V.Deksnys, "Approach to Diagnostics and Monitoring of Pneumatic Systems", Kaunas University of Technology- Radio equipment department, 2000.

[6] PHD of HAO BAI, "A generic fault detection and diagnosis approach for pneumatic and electric driven railway assets", 2010.

[7] Faisel J Uppal, Ron J Patton and Vasile Palade, "Neuro-fuzzy based fault diagnosis applied to an electro-pneumatic valve", Control \& Intelligent Systems Engineering, University of Hull, UK, 2002.

[8] Sang-Oak Song, Gibaek Lee and En Sup Yoon, "Process monitoring of an electropneumatic valve actuator using kernel principal component analysis", School of Chemical Engineering, Seoul National University, 2002 IFAC.

[9] MacGregor J.F., Yu H., Munoz S. and Cerrillo J., "Data-based latent variable methods for process analysis, monitoring and control", Journal of Computers and Chemical Engineering 29, 1217-1223, 2005.

[10] P. Nomikos and J. MacGregor, "Monitoring batch processes using multiway principal component analysis", AIChE J., Vol.40, 1361, 1994.

[11] Eriksson L., Johansson E., Wold N.K. and Wold S., "Multi- and Megavariate data analysis, principles and applications"; Umetrics AB, Sweden, 2006.

[12] Kourti T. and MacGregor J. F., "Multivariate SPC methods for process and product monitoring", Journal of quality technology, Vol.28, 4, October, 1996.

[13] Wold S., Esbensen K. and Geladi P., "Principal component analysis", Chemometrics and intelligent laboratory systems, 2: 37-52, 1987.

[14] Jackson J.E., “A User's Guide to Principal Components”, Wiley, New York, 1991.

[15] Wold S., Sjostrom M. and Eriksson L., "PLS-regression: a basic tool of chemometrics", Chemometric and intelligent laboratory systems, 58:109-130, 2001.

[16] Lennox B., Hiden H.G., Montague G.A., Kornfeld G. and Goulding P.R., "Application of multivariate statistical process control to batch operation", J. Computers and chemical engineering, V24, n2, July, PP. 291-296, 2000.

[17] Goulding P.R., Lennox B., Sandoz D.J., Smith K.J. and Marjanovic, "Fault detection in continuous processes using multivariate statistical methods", International journal of systems science, 31(11), pp. 1459-1471, 2000.

[18] Yon S., Landry J., Wold N.K., Pepe and Wold S., "Multivariate process monitoring and early fault detection (MSPC) using PCA and PLS", Plant automation and decision support conference, September 21-24, San Antonio, Texas, 2003.

[19] Chen J. and Liu K.C., "On-line batch process monitoring using dynamic PCA and dynamic PLS models", J. Chemical engineering science 57:63-75, 2002.

[20] Kourti T., "Abnormal situation detection, three-way data and projection methods robust data archiving and modeling for industrial applications", Annual reviews in control 27: 131-139, 2003.

[21] P. Nomikos and J. MacGregor, "Monitoring batch processes using multiway principal component analysis", AIChE J., Vol.40, 1361, 1994.

[22] Jolliffe, I.T., "Principle component analysis", springer virlag, New York, 1986.

[23] Leopold Simar and Wolfgang Hardle, “Applied Multivariate Statistical Analysis", Tech method and data technologies, Springer Verlag, Berlin and Louvain-la-Neuve, 2003. 